\section{Digestive \\ Surgergery}

Baeten, C.G.M.I. 150

Belghiti, J. 109

Bemelman, W.A. 147

Boermeester, M.A. 125,135

Bonavina, L. 86

Bosonnet, L. 138

Büchler, M.W. 123

Buskens, C.J. 88

Coevorden, F. van 88

Coosemans, W. 92

Decker, G. 92

De Leyn, P. 92
Dejong, C.H.C. 150

Delden, O.M. van 99

Fockens, P. 99

Ghaneh, P. 138

Gouma, D.J. 83, 114, 117

Gulik, T.M. van 106

Halloran, C.M. 138

Hartley, M.N. 138

Jeppsson, B. 105

Klar, E. 123

Lanschot, J.J.B. van 86, 88, 99

Leerdam, M. van 99
Lerut, T. 92

Marini, P. 109

McKay, C.J. 129

Nafteux, P. 92

Neoptolemos, J.P. 138

Obertop, H. 83, 88, 117

Soeters, P.B. 150

Sutton, R. 138

Van Raemdonck, D. 92

Vilgrain, V. 109

Williams, N.S. 150

Zoete, J.P.J.G.M. de 150

\title{
Subject Index Vol. 19, No. 2, 2002
}

Abdominal packing 106

Abscess drainage 150

Acalculous cholecystitis 125

Acute pancreatitis 125,129

Adenoma 109

Anastomotic leakage 135,150

- techniques 92

Antibiotics 125

Bile duct injury 114,117

- leakage 114, 117

Biliary strictures 114,117

Biloma 114, 117

Bleeding 99

Cholecystectomy 114, 117

- techniques 92

Colorectal 147

- surgery 150

Complications 135
Disconnection anastomosis 150

Diverticulitis 147

Diverting colostomy 150

Endoscopy 99

Esophageal leak 92

- reconstruction 92

- stenosis 92

Esophagectomy 88, 92

Fistula 88

Haemoperitoneum, rupture 109

Hartmann's procedure 147, 150

Hepatocellular adenoma 106

- carcinoma 109

Laparoscopic surgery 114,117

Liver bleed 106

- resection 106

- tumor 106

Minimally-invasive surgery 129
Morbidity 138

Mortality 138

Pancreatic cancer 138

- necrosis 125,129

- surgery 135

Pancreaticoduodenectomy 135

Peptic ulcer 99

Perforation 147

Post-operative complications 138

Primary anastomosis 150

Pseudocyst 135

Re-operation rate 138

Resection 138

Risk factors 150

Staged procedures 150

Surgery 99,125

Trachea 88

Treatment 125, 150

\section{KARGER}

(C) 2002 S. Karger AG, Basel

Fax + 41613061234

E-Mail karger@karger.ch

www.karger.com 